IHES/P/07/19

\title{
Wormholes as Black Hole Foils
}

\author{
Thibault Damour* and Sergey N. Solodukhin ${ }^{* \dagger}$ \\ * Institut des Hautes Etudes Scientifiques, \\ 35, route de Chartres, \\ 91440 Bures-sur-Yvette, France \\ $\dagger$ School of Engineering and Science, \\ International University Bremen, \\ Bremen 28759, Germany
}

\begin{abstract}
We study to what extent wormholes can mimic the observational features of black holes. It is surprisingly found that many features that could be thought of as "characteristic" of a black hole (endowed with an event horizon) can be closely mimicked by a globally static wormhole, having no event horizon. This is the case for: the apparently irreversible accretion of matter down a hole, no-hair properties, quasinormal-mode ringing, and even the dissipative properties of black hole horizons, such as a finite surface resistivity equal to $377 \mathrm{Ohms}$. The only way to distinguish the two geometries on an observationally reasonable time scale would be through the detection of Hawking's radiation, which is, however, too weak to be of practical relevance for astrophysical black holes. We point out the existence of an interesting spectrum of quantum microstates trapped in the throat of a wormhole which could be relevant for storing the information "lost" during a gravitational collapse.
\end{abstract}




\section{Introduction}

One of the most striking predictions of Einstein's theory of gravity is the existence of black holes. Though these objects made their first appearance in the famous exact spherically symmetric solution found by Karl Schwarzschild [1] a couple of months after Einstein finalized his theory, it took many years, and the work of many physicists, to cristallize the concept of black hole (see, e.g.,[2]). For a long time, a part of the physics community was rather sceptical about the actual existence of black holes, but the situation has changed in recent years, notably because of several different types of astronomical observations: in X-ray binary systems, in galactic nuclei (including our home, the Milky Way), etc. For a review of the astronomical evidence for black holes see [3].

Today black holes are part of the basic "toolkit" of physicists and astrophysicists, and their existence in the real universe is taken for granted. It is, however, interesting to examine critically to what extent the current, or future, astrophysical data can observationally prove the existence of black holes. Indeed, black holes are sophisticated theoretical constructs with many different properties and each observational evidence usually concerns only one specific property. For instance, in many observations the "black hole candidates" are mainly picked either because their inferred mass exceeds some theoretical limit, or on the basis of their strong external gravitational field. Several authors have claimed that some observations have probed, or will eventually probe, more characteristic features of black holes, and notably the (essentially defining) existence of an event horizon. For instance, Narayan and collaborators have argued that, in several examples, a black hole candidate "does not have a surface, i.e. it must have an event horizon" [3, 4]. In a different vein, it is also commonly argued that forthcoming gravitational wave data from LIGO/Virgo/GEO will establish the existence and "unique" properties of black holes either through the observation of the characteristic "quasi-normal mode" (QNM) ringing frequencies of a newly formed hole [5], or from observational checks of the "unique" structure of the black hole geometry guaranteed by "no-hair" theorems [6, 7].

A well-known, and useful strategy for gauging the extent to which observations can really characterize the presence of general relativistic black holes is to consider "black hole foils", i.e. theoretical objects that mimic some aspects of black holes, while lacking some of their defining features. Several examples of this strategy have been considered in the past. For instance, would-be black holes within Rosen's bimetric theory of gravity [8], or, more recently, some "gravstar" models [9].

In this note, we consider a very simple type of black hole foil: a wormhole [10]. Though a wormhole does not have an event horizon, and differs, in principle, in several other important ways from a black hole, we shall show here that, if a certain parameter entering its definition is small enough, a wormhole is essentially astrophysically indistinguishable from a black hole. Our final conclusion is that the possibly unique way of conclusively proving the presence of a black hole (endowed with an horizon) would be to observe its Hawking radiation [11]. And even this conclusion needs some qualification, because we shall see that some features of wormholes naturally tend to mimic the quantum spectrum of black holes, so that it is possible that some (to be defined) wormhole formation mechanisms could lead to an Hawking-like radiation. 


\section{$2 \quad$ Wormhole metric}

We shall consider here a very simple type of wormhole spacetime, as described by the metric

$$
d s^{2}=-\left(g(r)+\lambda^{2}\right) d t^{2}+\frac{d r^{2}}{g(r)}+r^{2}\left(d \theta^{2}+\sin ^{2} \theta d \phi^{2}\right),
$$

where $g(r) \equiv 1-\frac{2 G M}{r}$. This metric differs from the standard Schwarzschild metric [1] only through the presence of the dimensionless parameter $\lambda$. When $\lambda=0$ we recover a black hole of mass $M$ with an event horizon located at the radius $r=2 G M$. By contrast, when $\lambda \neq 0$ the structure of the spacetime is dramatically different: there is no event horizon, instead there is a throat at $r=2 G M$ that joins two isometric, asymptotically flat regions. This spacetime is an example of a Lorentzian wormhole [10]. In three dimensions a similar modification of the black hole metric was studied in [12] in an attempt to restore Poincaré recurrences in black holes. The parameter $\lambda$ in the latter construction was chosen to be exponentially small

$$
\lambda \sim e^{-4 \pi G M^{2}}
$$

in order to reproduce the expected dependence of the Poincaré recurrence time on the entropy of a black hole. Though we shall leave free the value of $\lambda$ in this paper, and discuss what range of values for $\lambda$ is compatible with present and foreseeable observations, we will see below that exponentially small values of the type of (2.2) seem indeed adequate for mimicking not only the classical, but also the quantum properties of a Schwarzschild black hole.

The event horizon of the original black hole metric is replaced, in the wormhole metric (2.1), by a high-tension distribution (a kind of brane) localized in a thin shell around the center of the throat at $r=2 G M$. More precisely, with our simplifying choice of wormhole metric (2.1), the stress-energy tensor distribution sustaining the throat has vanishing energy density, but comprises radial and tangential tensions proportional to $1 / \lambda^{2}$.

In order to define globally the wormhole spacetime (2.2) we need to specify how the spacetime is continued through the (geometrically regular) throat $r=2 G M$. The Schwarzschild-type radial coordinate $r$ is not well defined at $r=2 G M$. We should replace it, for instance, by the proper radial distance, say $y=\int_{2 G M}^{r} d r / \sqrt{g}$. In terms of $y$, one has, to leading order, the following expressions in the throat: $g(y)=\frac{y^{2}}{16 G^{2} M^{2}}$ and $r(y)=2 G M+\frac{y^{2}}{8 G M}$. Using the coordinate $y$ we can now globally define the wormhole spacetime in several different (physically inequivalent) ways. A first possibility (which is the usual one when considering "wormholes") is to decide that the variable $y$ varies over the full real line: $-\infty<y<+\infty$. A second possibility is to impose some $Z_{2}$ symmetry between $y$ and $-y$, so that $y$ effectively varies only on a half-line $0 \leq y<+\infty$ (with some $Z_{2}$-symmetry boundary conditions at $y=0$ ). We might prefer the first possibility if we have in mind a multi-brane-world in which the collapse of a star establishes a bridge between two previously separate brane-worlds. If, instead, we have in mind a unique world, we might prefer imposing the second possibility, i.e. the idea that the collapse of a star creates an "end-of-the-world" $Z_{2}$-symmetric brane at $r=2 G M$, which is certainly a logically allowed possibility. Note then that, in both cases, the wormhole spacetime (2.2) is globally static, the time Killing vector being everywhere timelike (while it became spacelike beyond the horizon in the black hole case). 
An immediate consequence of the metric (2.1) is that time in the throat is extremely slow from the point of view of a distant observer. Indeed, they are related by $\lambda$,

$$
t_{\mathrm{thr}}=\lambda t_{\mathrm{dist}}
$$

The throat thus mimics what happens at the event horizon of a black hole where time is "frozen" [we recall that the old name (especially in Russia) for a black hole was a "frozen star"]. The only difference from an actual horizon is that time does not completely stop in the throat: if an observer makes observations during a time of order $G M / \lambda$ he or she will resolve the processes happening in the throat and thus be able to distinguish a wormhole from a black hole. Reciprocally, this preliminary remark suggests that if an observer only looks at a wormhole during a finite time he or she might not be able to distinguish it from a black hole. We shall see below, in several examples, that this is indeed the case, even for phenomena that are usually considered as characteristically linked to the presence of an horizon (such as no-hair properties, or dissipative properties). However, we shall see that the observing time span needed to distinguish a wormhole from a black hole is not $G M / \lambda$, as suggested by the above naive argument, but rather $G M / \ln (1 / \lambda)$.

\section{Geodesics}

As first, and simplest example of the comparative phenomenology of wormholes versus black holes, let us consider the motion of particles around a wormhole, and their fall within the throat.

The (equatorial) geodesics in the metric (2.1) are described by the equations

$$
\begin{aligned}
& \dot{t}=\frac{E}{g(r)+\lambda^{2}}, \quad \dot{\phi}=\frac{L}{r^{2}}, \\
& \dot{r}^{2}+g(r)\left(\frac{L^{2}}{r^{2}}+\epsilon\right)=\frac{g(r)}{g(r)+\lambda^{2}} E^{2},
\end{aligned}
$$

where $\epsilon=0$ for a null-like geodesic, $\epsilon=1$ for a time-like geodesic, and where the overdot denotes the derivative with respect to the proper time (or an affine parameter, in the null case). $E$ is the energy and $L$ the angular momentum of the test particle (for simplicity

we consider a test particle of unit mass). In terms of the new coordinate $\rho=\int \sqrt{\frac{g+\lambda^{2}}{g}} d r$ the last equation in (3.1) takes the standard form

$$
\begin{aligned}
& \dot{\rho}^{2}+V(r(\rho))=E^{2}, \\
& V(r(\rho))=\left(g(r)+\lambda^{2}\right)\left(\frac{L^{2}}{r^{2}}+\epsilon\right) .
\end{aligned}
$$

The consideration of the "effective potential" $V(r)$ (or rather $V(r(y)$ ) to understand what happens in the throat) then allows one to understand qualitatively the dynamics of particles in the wormhole.

As soon as one is a little bit away from the throat, the dynamics is that for the Schwarzschild metric plus (observationally negligible) small corrections proportional to $\lambda^{2}$. This shows that any observational feature which is not taking place very near $r=2 G M$ will be (for small enough $\lambda$ ) the same in the wormhole foil than in a real black hole. This is for instance the case for the emissivity properties of accretion disks, even those 
that crucially depend on relativistic dynamics features (like the presence of an innermost stable circular orbit (ISCO)).

On the other hand, there is an important difference from the black hole case if we consider, say, circular orbits with radius equal to (or very near) the throat radius $r=2 G M$. Both for null and timelike geodesics, and for any value of the angular momentum $L 11$ there exists a circular orbit exactly located at $r=2 G M$. The energy and angular momentum in this case are related by $E^{2}=\lambda^{2}\left(\frac{L^{2}}{4 G^{2} M^{2}}+\epsilon\right)$. The derivative of the radial potential vanishes $\partial_{\rho} V=0$ for $r=2 G M$ while the second derivative $\left.\partial_{\rho}^{2} V(\rho)\right)=\left.\frac{1}{2 \lambda^{2}} V_{r}^{\prime} g_{r}^{\prime}\right|_{r=2 G M}$ is positive. Thus, this "throat-orbiting" circular orbit is stable. In addition, the positive curvature of the effective potential at $r=2 G M$ implies that there exist bound "elliptic" orbits staying near $r=2 G M$.

An argument often evoked for distinguishing a black hole from other potential wells is the absence of a "surface" in the black hole case, and the possibility for the horizon of absorbing any amount of infalling matter [3, 4]. The situation is a priori quite different in the wormhole case because a look at the qualitative shape of the effective potential $V(r(y))$ shows that, for instance, particles falling from (just below) the ISCO must ultimately bounce back up again from the wormhole throat 2

To study in more detail this "bounce" from a wormhole, let us focus for simplicity on the case of radial timelike geodesics, with $L=0$ and therefore $V(r)=\epsilon\left(g(r)+\lambda^{2}\right)$ (with $\epsilon=1)$. There are 3 cases. If $E^{2}>1+\lambda^{2}$ then a particle coming from infinity falls into the wormhole. These geodesics are similar to the radial geodesics in the Schwarzschild metric. If $\lambda^{2}<E^{2}<1+\lambda^{2}$ then the geodesic has a turning point $y=y_{m}$ in which $\dot{y}=0$. The coordinate of the turning point must solve the equation $g\left(y_{m}\right)+\lambda^{2}=E^{2}$. There are exactly two solutions to this turning-point equation, a positive one $y_{m}>0$ and its opposite $-y_{m}<0$. If the wormhole connects two separate spaces, these turning points are physically distinct, and the radial geodesic bounces back, in an oscillatory manner, between them. In the $Z_{2}$-symmetric case, the radial geodesic bounces between the positive turning point and $y=0$ (with half the period taken in the former case). There is no analog of these geodesics in the case of the Schwarzschild metric. Finally, if $E^{2}<\lambda^{2}$ there is no solution to the geodesic equation. Note also that for null radial geodesics( $L=0, \epsilon=0$, so that the effective potential vanishes) there are no oscillating solutions in the two-separate-spaces case, the light irreversibly falls into the wormhole as it does in the black hole. But, in the $Z_{2}$-symmetric case, light bounces back at the throat and exits from the mouth of the wormhole.

All this suggests that present observations of accreting (or formerly accreting) gravitational potential wells rule out their modelling as wormholes. However, as anticipated above from the basic scaling (2) it is crucial to study on what time scale the matter which falls within a wormhole does come out again in our universe. Let us compute the coordinate time taken by a particle $(\epsilon=1)$ to go (by geodesic motion) from a point outside the wormhole, $y=l>0$ to a point inside the throat (say $y=0$ ). The same calculation, but now taken for $\epsilon=0$, will give the coordinate time taken by a light signal to join a point inside the throat (say $y=0$ ) to a point outside the wormhole $y=l>0$. These coordinate times are given by

\footnotetext{
${ }^{1}$ including $L=0$, in which case one has an equilibrium position at fixed $r$ and $\phi$.

${ }^{2}$ This conclusion holds in the case where $y$ ranges over the full real line (in which case the effective potential is made of two $y$-symmetric humps), as well as in the $Z_{2}$-symmetric case where the effective potential has only one hump, but where the particle bounces off when it reaches $y=0$.
} 


$$
\begin{array}{ll}
\Delta t=\int_{0}^{l} \frac{E d y}{\sqrt{\left(g+\lambda^{2}\right)\left(E^{2}-\lambda^{2}-g\right)}}, \epsilon=1 \\
\Delta t=\int_{0}^{l} \frac{d y}{\sqrt{g+\lambda^{2}}}, & \epsilon=0
\end{array}
$$

Irrespectively of the type of the geodesic this time is dominated by the throat region, i.e. the part of the integrals where $g(y) \sim \lambda^{2}$. As both integrals are logarithmically divergent in the black hole limit, $\lambda \rightarrow 0, \Delta t \sim \int d y / \sqrt{g} \sim \int d r / g(r)$, it is easily seen that the leading term when $\lambda \neq 0$ is

$$
\Delta t=2 G M \ln \frac{1}{\lambda^{2}}
$$

This result shows that if $\lambda$ is small enough, it is impossible for observations extending on some limited time scale $T$ to distinguish the provisory fall of matter in a wormhole from the irreversible absorption of matter down the horizon of a black hole. For instance, if we consider the candidate "massive black hole" ( with $M \sim 3 \times 10^{6} M_{\odot}$ ) at the center of our Galaxy, and assume it started accreting matter 6 billion years ago, it could be a wormhole if $\lambda \ll \exp \left(-10^{15}\right)$.

We clearly need exponentially small values of $\lambda$ to mimic observational facts. Note that this would be precisely the case if we were using the value (2.2) above, as suggested for quite different reasons in [12. Actually, if we substitute here the value (2.2) for the parameter $\lambda$ in the "wormhole bounce time scale" (3.4) we get

$$
\Delta t=16 \pi G^{2} M^{3}
$$

which is of the order of the Hawking evaporation time scale for a Schwarzschild black hole. We shall come back below to this suggestive link between quantum black holes and (classical and/or quantum) wormholes.

Before discussing other phenomenological aspects of wormholes, let us mention a potential difficulty with the wormhole model proposed here. In this paper we shall content ourselves with a first-order treatment in which the matter and fields "falling into" a wormhole are considered as test-matter propagating in a given wormhole background. However, the stress-energy tensor carried by all the matter that have accreted in the past onto a wormhole (and that is, for all practical purposes, essentially "frozen" around the throat $r=2 G M)$ will distort the background wormhole metric. However, it is well known [10] that a wormhole requires that the "matter" making it up must violate (some form of) the positive-energy condition (we saw that above in the fact that the energy density corresponding to the metric (2.1) vanishes, while the tension does not vanish). As the accreted matter does satisfy the usual positive energy conditions, it is not clear how much accreted matter can be allowed in before risking to destroy the wormhole throat. Actually, we should provide a more complete definition of our wormholes as dynamical objects. For instance, one should, in principle, discuss the dynamical structure of the "brane" located at $y=0$, and its possible interaction with the matter falling onto it. Even without such a complete dynamical definition, we think that it is interesting to explore, as we do here, how wormholes can be conceptually clarifying foils for black hole dynamics. 


\section{$4 \quad$ No-hair properties}

As an example of the way wormholes can mimic the no-hair properties of black holes, let us consider static axisymmetric (test) electric fields in a general wormhole background $d s^{2}=$ $-A(r) d t^{2}+B(r) d r^{2}+r^{2}\left(d \theta^{2}+\sin ^{2} \theta d \phi^{2}\right)$. The static Maxwell equations, $\partial_{\nu}\left(\sqrt{-g} F^{\mu \nu}\right)=0$ reduce (when taking $\mu=t$ ) to a second-order differential equation (in $r$ and $\theta$ ) for the electric potential $A_{t}$. One easily separates the $r$ and $\theta$ dependence by factoring: $A_{t}(r, \theta)=$ $a(r) P_{l}(\cos \theta)$, where $P_{l}(\cos \theta)$ is a usual Legendre polynomial. This leads to the following separated equation for the radial factor $a(r)$

$$
\sqrt{\frac{A}{B}} \partial_{r}\left(\frac{r^{2}}{\sqrt{A B}} \partial_{r} a(r)\right)=l(l+1) a(r) .
$$

Let us consider, for example, the wormhole metric $A=g+\lambda^{2}, B=1 / g$, taken in the $Z_{2^{-}}$ symmetric case. One generically sees that a solution which is regular and $Z_{2}$-symmetric in the throat ( $d a / d y=0$ at $y=0)$ will grow like $r^{l}$ at infinity. Therefore we indeed have a no-hair property paralleling the one for black holes: the only solution which is regular at the throat, and decaying at infinity, is the trivial one $a(r)=0$ for any $l 3$.

From this no-hair property one deduces that if one brings a point charge near the throat $r=2 G M$ (but parametrically far away from the $r-2 G M \sim 2 G M \lambda^{2}$ "near-throat limit") this will generate an electric field which is essentially indistinguishable (modulo corrections $\propto \lambda^{2}$ ) from the one generated near a black hole, i.e. an electric field which, when seen from outside, erases the information about the location of the point charge and looks like a spherically symmetric electric field centered on the hole (see [13]).

\section{Quasi-normal mode ringing}

It is often said that the observation by the LIGO/Virgo/GEO network of gravitational wave detectors of the quasi-normal mode (QNM) ringing of a newly formed (rotating) black hole will provide an excellent confirmation of the actual existence of black holes in Nature [5]. Indeed, the definition of QNM modes depends in a crucial way on the presence of an event horizon. Let us recall that the QNM modes are defined as complex-frequency eigenmodes which satisfy the boundary conditions of being outgoing at radial infinity, and ingoing towards the black hole horizon. To discuss what happens of QNM modes in a wormhole background let us consider, for simplicity, the case of scalar field modes. [Our physical discussion will make it clear that our conclusions apply to the more relevant (tensor) gravitational excitations.]

For a mode of a scalar field of frequency $\omega, \Phi=\frac{1}{r} \psi(r) e^{-i \omega t} Y_{l m}(\theta, \phi)$, we get a radial equation in the Regge-Wheeler-Zerilli form, i.e. an effective Schroedinger equation

$$
\begin{aligned}
& -\psi_{z z}+U(z) \psi=\omega^{2} \psi, \\
& U(z)=\frac{r_{z z}}{r}+\frac{l(l+1)}{r^{2}}\left(g(r)+\lambda^{2}\right) .
\end{aligned}
$$

Here, a $z$ subscript denote a $z$ derivative, $l=0,1,2, \ldots$ and we have used as variable the "tortoise" radial coordinate $z=\int_{2 G M} d r \sqrt{\frac{B}{A}}=\int_{2 G M} \frac{d r}{\sqrt{g\left(g+\lambda^{2}\right)}}$, which is usually denoted $r_{*}$,

\footnotetext{
${ }^{3}$ In the non- $Z_{2}$-symmetric case, the monopolar case, $l=0$, is a special case in that there exists a source-free everywhere regular solution parametrized by a charge $Q$, namely $\partial_{y} a(r)=\frac{Q \sqrt{A}}{r^{2}}$.
} 
and which is such that the radial part of the metric $\left(d s^{2}=-A d t^{2}+B d r^{2}\right)$ is conformal to $-d t^{2}+d z^{2}$. Inserting $r_{z z}=g_{r}^{\prime}\left(g+\frac{\lambda^{2}}{2}\right)$, we get the following explicit form for the effective radial potential

$$
U(r)=\frac{2 G M}{r^{3}}\left(1+\frac{\lambda^{2}}{2}-\frac{2 G M}{r}\right)+\frac{l(l+1)}{r^{2}}\left(1-\frac{2 G M}{r}+\lambda^{2}\right) .
$$

For a wormhole connecting two separate spaces, the tortoise radial coordinate $z$ varies over the full real line, and this potential is made of two separate positive humps located at the positive and negative values of $z$ corresponding to $r \sim 3 G M$. In the $Z_{2}$-symmetric case we have only one hump, together with a suitable boundary condition at the throat located at $z=0$. Let us think in terms of the easily visualizable two-humped potential. [The $Z_{2}$-symmetric case consists anyway in retaining the $z$-even solutions of the other case.]

This two-humped potential clearly has a very different spectrum than the usual black hole effective potential which has only one positive hump located around $r \sim 3 G M$. If we look for modes satisfying the usual QNM condition of being purely outgoing (both towards $z \rightarrow+\infty$ and towards $z \rightarrow-\infty$ ) we will have a spectrum which is qualitatively very different from the usual black hole QNM spectrum. Indeed, it will now contain modes with a real part of the frequency lower than the maximum of the effective potential, and a very small imaginary part. These modes are quasi-bound states ("resonances") trapped within the two-humped potential, with a small escape probability and a long lifetime. There is no analog of these modes in the black hole case. As for the former black hole QNM modes, they do not seem to play any prominent role anymore. Indeed, near, but on the left of the rightmost hump, there will exist, for a general "wormhole QNM mode", a combination of left-moving and right-moving modes which has nothing to do with the black hole QNM modes which are purely left-moving (i.e. away from the summit of the potential).

Have we got here a clear observable distinction between a wormhole and a black hole? In fact not. Indeed, the observable way in which one hopes to detect QNM ringing in the black hole case consists in considering the signal emitted by a source falling into the hole, i.e. a source starting at some large and positive value of $z$, and moving leftwards towards smaller values of $z$. The observable signal emitted by this source is obtained, in the time domain, by the convolution of the retarded Green function $G_{\text {ret }}\left(t-t^{\prime}, z, z^{\prime}\right)$ corresponding to the Klein-Gordon-like (time-domain) equation (5.1) with the source, say $\delta\left(z^{\prime}-z_{\text {geodesic }}\left(t^{\prime}\right)\right)$. It is true that the wormhole retarded Green function $G_{\text {ret }}\left(t-t^{\prime}, z, z^{\prime}\right)$ is globally quite different in the wormhole spacetime, compared to the black hole case, because there will be a combined diffusion effect due to the two potential humps. However, if the observer looks at the emitted signal only over time scales much smaller than the time it takes for a causal signal to go from a source event $\left(t^{\prime}, z_{\text {geodesic }}\left(t^{\prime}\right)\right)$ [located, say, near the rightmost hump] to the leftmost hump, and then to scatter back to the right until the observation event $(t, z)$, the observed signal will be the same as that computed by using only the diffusion effect of the rightmost hump, i.e. the retarded Green function of a black hole spacetime. And this computation, when done in the Fourier domain, will exhibit phenomena linked to the usual black hole QNM modes. This indirect, but physically clear reasoning, shows that if $\lambda$ is such that the time scale (3.4) is longer than the observational time scale the signals emitted by a source falling into a wormhole will contain the usual QNM ringing "signature of a black hole", in spite of the absence of a 
true horizon in the wormhole case.

\section{Dissipative properties}

Let us now discuss whether wormholes can mimic the dissipative properties of black holes, and notably the fact that they can be described as membranes having a finite electric (surface) resistivity equal to 377 Ohms [14] (as well as a finite (surface) viscosity [15]). Indeed, the proof of these properties crucially relies on the presence of an horizon.

We might have here a good way of observationally discriminating wormholes from black holes. For instance, we can consider the physical situation discussed in [14]. An electric current $I$ is passed through a black hole, penetrating through the North pole and exiting from the South polet. This generates a certain stationary electromagnetic field. The analysis in [14] of the regularity of the field structure on the event horizon has shown that (even for a non-rotating hole) the magnetic field generated by the current must be accompanied by a correlated electric field. It was then explicitly verified that the latter electric field is responsible for generating an electric potential difference ( an "EMF") between the two poles such that Ohms' law $V=R I$ is satisfied, with a resistance $R \sim 30$ Ohms computable "as if" the black hole horizon were a conducting surface of resistivity equal to $377 \mathrm{Ohms}$ (i.e. $4 \pi$ ). This electric potential difference is, in principle, observable, and might actually play a significant role in magnetic-field based mechanisms for extracting energy from black holes [16, 17], which are believed to be important in active galactic nuclei and other astrophysical processes.

If we pass a current $I$ through a wormhole, we expect, at face value, to generate only a magnetic field. More precisely, adopting the geometry of current injection of [14], and solving Maxwell equations for a general spherically symmetric wormhole background, we find a solution involving a purely magnetic field strength

$$
F^{\text {wormhole }}=d A=\frac{2 I}{\sin \theta} \sqrt{\frac{B}{A}} d \theta \wedge d r .
$$

This is consistent with [14], but represents only the magnetic part of the result

$$
F^{\text {blackhole }}=d A=\frac{2 I}{\sin \theta} d \theta \wedge\left(d t+\frac{d r}{1-2 G M / r}\right)
$$

If that were all, the difference between the last two results would signal a clear physical distinction between a wormhole and a black hole. However, (6.1) has been derived by looking for a stationary solution of Maxwell equations in a wormhole background. This would be reasonable for a usual object through which passes a stationary current. But a wormhole is not a usual object, and we must take into account the crucial physical fact which played an important role above. When $\lambda$ is very small, one must remember that far from leading to a stationary state the charges (of opposite signs) continuously sent onto the poles of a wormhole will appear (on usual external timescales) to accumulate on the North and South poles of the $r=2 G M$ throat, though the effect of these localized

\footnotetext{
${ }^{4}$ This can be realized by sending a flux of positive charges through the North pole, and a flux of negative charges through the South pole.
} 
accumulated charges will tend to uniformly spread out into a low-multipole electric field. When thinking more about this rather complicated problem, one then realizes that we can use the same arguments that we have used already above. It consists essentially in saying that the retarded Green function (now considered for a Maxwell field) in a wormhole spacetime will precisely mimic (if $\lambda$ is small enough) the black-hole retarded Green function if one considers a source which started falling in a finite time ago, and an observer having also a finite observing time window.

\section{$7 \quad$ Quantum effects}

Let us finally consider quantum effects in a wormhole metric, and compare them to the black hole case.

We have already seen that in a wormhole spacetime there are classical geodesics, absent in the Schwarzschild metric, which oscillate around the throat region. We therefore expect that there will be corresponding quantum modes which are trapped within the throat. Actually, we have already mentioned them above. Indeed, when considering, for simplicity, a quantum scalar field $\Phi$ propagating in a wormhole metric, we can decompose it in modes of frequency $\omega$ and angular momentum $(l, m)$. This leads to the separated radial equation written in equation (5.1) above.

The effective radial potential (5.2) reaches a minimum (positive) value min $U=$ $\frac{\lambda^{2}}{4 G^{2} M^{2}}\left[\frac{1}{2}+l(l+1)\right]$ at the center of the throat $r=2 G M$. Note that this minimum value is positive, but tends to zero like the square of $\lambda$. This minimum is surrounded on both sides by much higher positive maxima located around (we take the limit $l \gg 1$ in which these expressions simplify) $r=\frac{3 G M}{1+\lambda^{2}}$ and of value

$$
\max U=\frac{1}{27 G^{2} M^{2}}\left(l(l+1)+\frac{2}{3}\right)+\frac{1}{9 G^{2} M^{2}}\left(l(l+1)+\frac{5}{9}\right) \lambda^{2} .
$$

As we already mentioned above when discussing QNM modes, the radial equation (5.1) admits a discrete set of long-lived resonances within this potential well. The lowest (nearly real) energy levels can be obtained by looking in the throat region $r-2 G M \ll$ $2 G M$. There the relation between the coordinates $r$ and $z$ is given by $r(z)=2 G M(1+$ $\lambda^{2} \sinh ^{2} \frac{z}{4 G M}$ ). The effective radial potential takes the form (for arbitrary $l$ )

$$
U(z)=\frac{\lambda^{2}}{8 G^{2} M^{2}}(1+2 l(l+1))+\frac{\lambda^{2}}{4 G^{2} M^{2}}(1+l(l+1)) \sinh ^{2}\left(\frac{z}{4 G M}\right) .
$$

The discrete spectrum for this potential can be obtained in the WKB approximation [12],

$$
\omega_{n}=\frac{\pi}{8 G M \ln (1 / \tilde{\lambda})}\left(n+\frac{1}{2}\right), n \in Z
$$

where we defined $\tilde{\lambda}^{2}=\lambda^{2}(1+l(l+1))$ and neglected terms of order $\lambda^{2}$ (and $\left.\tilde{\lambda}^{2}\right)$.

For $l=0$ the first discrete level appears far above the bottom of the potential well $\frac{\lambda^{2}}{8 G^{2} M^{2}}$ but far below the top of the potential. Thus, there is a large number $\mathcal{N}_{0}$ of discrete levels inside this $l=0$ well. This number can be estimated as

$$
\mathcal{N}_{0} \simeq \frac{\sqrt{27}}{4 \pi} \ln \frac{1}{\lambda}
$$


If we consider the case where $\lambda$ is given by (2.2), we note that the number of bound states is of the order of $\mathcal{N}_{0} \sim G M^{2}$, which is of the same order as the entropy of the Schwarzschild black hole. Note, however, that this was only the $l=0$ modes. If we consider $l \neq 0$ modes we will have a similar spectrum of quasi-bound states, and the number of bound states for given values of $l$ and $m$ will be of order (for large $l$ and neglecting $\lambda^{2}$ terms)

$$
\mathcal{N}_{l m} \simeq \frac{8}{\pi \sqrt{27}} \sqrt{l(l+1)+\frac{2}{3}} \ln (1 / \tilde{\lambda}) \sim \sqrt{l(l+1)+\frac{2}{3}} G M^{2}
$$

It would be interesting to study more carefully whether these quasi-bound states could be considered as analogs of the somewhat mysterious "black hole microstates" which are supposed to be counted by the Bekenstein-Hawking entropy (see e.g. [18] for a review). Even more interestingly, as the wormhole resonances discussed here are all unstable, it is tempting to conjecture that they might somehow mimic the Hawking radiation. We have in mind here a mechanism of the following sort. During the collapse leading to the (assumed) formation of a wormhole, the quantum field $\Phi$ will be left in a state where many of the wormhole resonance modes will be excited. The modes which have a large decay width $\Gamma=-\Im \omega$ (like the ordinary QNM modes) will be radiated quite fast. But the modes which are deep down within the double-humped potential well will have a very small decay width $\Gamma$ and will slowly trickle out of the potential well, thereby generating a nearly continuous radiation emitted by the wormhole.

However, though we anticipate that this "wormhole radiation" might (especially for the choice (2.2) of $\lambda$ ) coarsely model Hawking's radiation, we do not think that it will be possible to reproduce with precision the specific thermal-like grey-body spectrum predicted in [11]. Indeed, this spectrum is a delicate consequence of the fact that the modes of a quantum field which "straddle" the event horizon get torn into two outgoing modes, one of which exits at radial infinity in the form of a quasi-thermal spectrum.

\section{Conclusions}

In conclusion we considered a wormhole spacetime as a "foil" to a Schwarzschild black hole, to learn to what extent the observational features of a black hole do really depend on the presence of an event horizon. Indeed, unlike a black hole, a wormhole geometry is globally static and does not have an event horizon. It was clear from the start that, as the two spacetimes have a nearly identical geometry for $r>2 G M$, they would have very similar closed geodesics, and would therefore be practically indistinguishable in astronomical observations that depend only on the external gravitational field. However, and more surprisingly, we found that many observational features that were thought to crucially depend on the presence of an event horizon were well mimicked by a wormhole, if the parameter $\lambda$ is sufficiently (exponentially) small. This includes, the apparently irreversible accretion of matter down a hole, no-hair properties, quasi-normal-mode ringing, and even the dissipative properties of black hole horizons, such as a finite surface resistivity equal to 377 Ohms [14].

Finally, we conclude that the only ways to observationally distinguish a wormhole from a black hole are: (1) either to observe classical phenomena (such as matter accretion) over the long "wormhole bounce" time scale $\Delta t=2 G M \ln \frac{1}{\lambda^{2}},(2)$ or to observe the Hawking radiation presently coming out of a hole. Interestingly, there is a link between these two 
methods: when $\lambda$ takes the value (2.2) suggested in 12 the classical wormhole bounce time scale becomes comparable to the quantum evaporation time of a Schwarzschild black hole $\Delta t=16 \pi G^{2} M^{3}$. However, in the case where $\lambda$ takes the value (2.2) both methods are unpractical because the time scale $\Delta t=16 \pi G^{2} M^{3}$ is much too large for usual astrophysical masses, and/or the Hawking temperature is much too low (being much smaller than the $3 \mathrm{~K}$ cosmological background).

It remains interesting to keep in mind that most of the phenomenology of black holes does not really depend on the presence of an horizon, and also (though this deserves more study) that a wormhole could somehow mimic the Hawking radiation, as well as may provide a simple way of visualizing the microstates storing the information apparently "lost" during a gravitational collapse. One would, however, need a more detailed model of the formation of a wormhole to address this issue.

Acknowledgments: SS would like to thank V. Frolov, T. Jacobson and D. Lowe for useful conversations at earlier stages of this work. SS is grateful to the IHÉS for the hospitality extended to him while this work was in progress.

\section{References}

[1] K. Schwarzschild, "On The Gravitational Field Of A Mass Point According To Einstein's Theory," Sitzungsber. Preuss. Akad. Wiss. Berlin (Math. Phys. ) 1916 (1916) 189; see English translation in arXiv:physics/9905030.

[2] C. W. Misner, K. S. Thorne and J. A. Wheeler, "Gravitation," San Francisco 1973, $1279 p$

[3] R. Narayan, "Black Holes in Astrophysics," New J. Phys. 7, 199 (2005) arXiv:gr-qc/0506078.

[4] A. E. Broderick and R. Narayan, "On The Nature of the Compact Dark Mass at the Galactic Center," Astrophys. J. 638, L21 (2006) arXiv:astro-ph/0512211.

[5] K. D. Kokkotas and B. G. Schmidt, "Quasi-normal modes of stars and black holes," Living Rev. Rel. 2, 2 (1999) arXiv:gr-qc/9909058.

[6] F. D. Ryan, "Gravitational waves from the inspiral of a compact object into a massive, axisymmetric body with arbitrary multipole moments," Phys. Rev. D 52, 5707 (1995).

[7] S. A. Hughes, "Trust but verify: The case for astrophysical black holes," in: Proceedings of 33rd SLAC Summer Institute on Particle Physics (SSI 2005): Gravity in the Quantum World and the Cosmos, Menlo Park, California, 25 Jul - 5 Aug 2005, pp L006 [arXiv:hep-ph/0511217].

[8] W. R. Stoeger, "Orbital Topography And Other Astrophysical Consequences Of Rosen's Bimetric Theory Of Gravity," Gen. Rel. Grav. 9, 165 (1978). 
[9] A. E. Broderick and R. Narayan, "Where are all the gravastars? Limits upon the gravastar model from accreting black holes," Class. Quant. Grav. 24, 659 (2007) arXiv:gr-qc/0701154.

[10] M. Visser, "Lorentzian wormholes: From Einstein to Hawking," Woodbury, USA: AIP (1995) $412 p$

[11] S. W. Hawking, "Particle Creation By Black Holes," Commun. Math. Phys. 43, 199 (1975) [Erratum-ibid. 46, 206 (1976)].

[12] S. N. Solodukhin, "Restoring unitarity in BTZ black hole," Phys. Rev. D 71, 064006 (2005) arXiv:hep-th/0501053; "Can black hole relax unitarily?," arXiv:hep-th/0406130.

[13] R. S. Hanni and R. Ruffini, "Lines of Force of a Point Charge near a Schwarzschild Black Hole," Phys. Rev. D 8, 3259 (1973).

[14] T. Damour, "Black Hole Eddy Currents," Phys. Rev. D 18, 3598 (1978).

[15] T. Damour, "Surface effects in black hole physics", in: Proceedings of the Second Marcel Grossmann Meeting on General Relativity", edited by R. Ruffini (NorthHolland, Amsterdam,1982), pp. 587-608.

[16] R. Ruffini and J. R. Wilson, "Relativistic Magnetohydrodynamical Effects Of Plasma Accreting Into A Black Hole," Phys. Rev. D 12, 2959 (1975).

[17] R. D. Blandford and R. L. Znajek, "Electromagnetic Extractions Of Energy From Kerr Black Holes," Mon. Not. Roy. Astron. Soc. 179, 433 (1977).

[18] T. Damour, "The entropy of black holes: A primer," in: Poincaré Seminar 2003, edited by J. Dalibard, B. Duplantier and V. Rivasseau (Birkhäuser Verlag, Basel, 2004), pp.227-264 arXiv:hep-th/0401160. 